\title{
BMJ Open Burden of non-accidental mortality attributable to ambient temperatures: a time series study in a high plateau area of southwest China
}

\author{
Changyu Deng, ${ }^{1}$ Zan Ding, ${ }^{2}$ Liujiu Li, ${ }^{3}$ Yanfang Wang, ${ }^{3}$ Pi Guo, ${ }^{1}$ Shaoyi Yang, ${ }^{1}$
} Ju Liu, ${ }^{1}$ Yue Wang, ${ }^{1}$ Qingying Zhang ${ }^{1}$

To cite: Deng C, Ding Z, Li L, et al. Burden of non-accidental mortality attributable to ambient temperatures: a time series study in a high plateau area of southwest China. BMJ Open 2019;9:e024708. doi:10.1136/ bmjopen-2018-024708

- Prepublication history and additional material for this paper are available online. To view these files, please visit the journal online (http://dx.doi. org/10.1136/bmjopen-2018024708).

Received 12 June 2018 Revised 21 December 2018 Accepted 2 January 2019

Check for updates

(C) Author(s) (or their employer(s)) 2019. Re-use permitted under CC BY-NC. No commercial re-use. See rights and permissions. Published by BMJ.

${ }^{1}$ Department of Preventive Medicine, Shantou University Medical College, Shantou, China

${ }^{2}$ The Institute of Metabolic Diseases, Baoan Central Hospital of Shenzhen, the Fifth Affiliated Hospital of Shenzhen University, Shenzhen, China

${ }^{3}$ Yuxi Center for Disease Control and Prevention, Yuxi, China

Correspondence to Professor Qingying Zhang; qyzhang@stu.edu.cn

\section{ABSTRACT}

Objective To examine the total non-accidental mortality burden attributable to ambient temperatures and assess the effect modification of the burden by specific causes of death and individual characteristics in a high plateau area in southwest China.

Methods Using daily mortality and meteorological data from 2009 to 2016, we applied a quasi-Poisson model combined with a distributed lag non-linear model to estimate the temperature-mortality association with the assessment of attributable fraction and number. We calculated attributable fractions and deaths with 95\% empirical Cls (eCls), that were due to cold and heat, defined as temperatures below and above the median temperature, and for mild and extreme temperatures, defined by cut-offs at the 2.5th and 97.5 th temperature percentiles.

Results We analysed 89467 non-accidental deaths; 4131 were attributable to overall temperatures, with an attributable fraction of $4.75 \%(95 \% \mathrm{eCl} 2.33 \%$ to $6.79 \%)$. Most of the mortality burden was caused by cold (4.08\%; $0.86 \%$ to $7.12 \%)$, whereas the burden due to heat was low and non-significant $(0.67 \% ;-2.44 \%$ to $3.64 \%)$. Extreme cold $(1.17 \% ; 0.58 \%$ to $1.69 \%)$ was responsible for $24.6 \%$ (ie, $1.17 \%$ divided by $4.75 \%$ ) of the total death burden. In the stratification analyses, attributable risk due to cold was higher for cardiovascular than respiratory disease (6.18\% vs $3.50 \%)$. We found a trend of risk of increased death due to ambient temperatures with increasing age, with attributable fractions of $1.83 \%, 2.27 \%$ and $6.87 \%$ for age $\leq 64,65-74$ and $\geq 75$ years old, respectively. The coldrelated burden was slightly greater for females, farmers, ethnic minorities and non-married individuals than their corresponding categories.

Conclusions Most of the burden of death was attributable to cold, and specific causes and individual characteristics might modify the mortality burden attributable to ambient temperatures. The results may help make preventive measures to confront climate change for susceptible population in this region.

\section{INTRODUCTION}

With the global climate change, ambient temperature has been extensively demonstrated to directly affect human health
Strengths and limitations of this study

- Mortality burden attributable to ambient temperature was assessed in a high plateau city in southwest China.

- To our knowledge, this study evaluated the mortality burden attributable to ambient temperature and quantified its effect modification by national minority and occupation for the first time.

- The data only come from one city, so it should be cautious to generalise the findings to other geographical areas or climates.

- We used the data on temperature from monitoring sites rather than measuring individual exposure, which may bring about measurement errors.

(eg, daily morbidity and mortality) and has become one of the most severe public health problems in the world. ${ }^{1-5}$ Exposure to extreme weather conditions such as cold spells and heat waves represents high risk for mortality, and the extreme temperature-related mortality is expected to increase with the increasing frequency, intensity and duration of extreme weather events. ${ }^{46-8}$ Low and high temperatures are also well known to be associated with a substantial increase in a wide range of all-cause and cause-specific mortality (eg, cardiovascular and respiratory diseases). ${ }^{6-12}$

Numerous epidemiological studies have widely used ratio measures (eg, OR, relative ratio or rate ratio) to quantify the relationships between ambient temperatures and human health, but these offer limited information on the excess burden and actual impact of ambient temperatures. ${ }^{13-16}$ Relative excess measures (eg, attributable fraction) and absolute excess measures (eg, attributable number), calculated on the basis of the estimated relative risk, have been pointed out to provide better scientific evidence 
for estimating the potential benefits of preventative measures, public health interventions and resource allocation. ${ }^{511} 17$ The attributable fraction and number represent the fraction and number of cases or deaths from a cause-specific disease that would be prevented without exposure to a specific risk factor, which has important implications for policymaking and the potential impact of interventions. ${ }^{18-20}$

The use of risk assessment of the attributable fraction revealed the burden of mortality associated with ambient temperatures; however, most previous literatures estimated the mortality burden in high-income or low-altitude regions or coastlands, ${ }^{5} 111921-23$ and few were conducted in high plateau areas of developing countries. ${ }^{12}{ }^{24}$ The attributable fraction and number for the temperaturemortality association may vary by geographical features, climate and structure of the population. ${ }^{24}{ }^{25}$ In addition, age, gender, educational attainment and specific causes were previously identified as modifiers for estimating the effect modification of the mortality risk attributable to ambient temperatures. ${ }^{26-30}$ However, few researchers have focused on the potential effect modification of the mortality burden by occupation, race/ethnicity or marital status. $^{31}$

Yuxi city is located in a high-altitude area in southwest China and experiences a unique, subtropical, plateau monsoon climate. More than $70 \%$ of indigenous people in this multi-ethnic region engage in agricultural production. The aim of this current ecological dissertation in Yuxi was to quantify the burden of non-accidental mortality attributable to ambient temperatures. We aimed to separate the contribution of temperature to mortality by heat and cold and mild and extreme temperatures by using attributable fraction and number, based on a proposed framework of attributable risk assessment within a distributed lag non-linear model (DLNM). A more in-depth purpose was to comprehensively assess the effect modification of the non-accidental mortality burden attributable to ambient temperatures by specific mortality causes (ie, cardiovascular, heart, stroke and respiratory diseases) and individual characteristics (ie, age, gender, occupation, ethnicity and marital status).

\section{METHODS}

\section{Study site}

Located on the western edge of the Yunnan-Guizhou Plateau of southwest China, the Yuxi city area has complicated geographical features of mountains, valleys, plateaus and basins. With an average altitude of about $2000 \mathrm{~m}$ and four spring-like seasons, this area has a unique, subtropical, plateau monsoon climate, showing diversified climates with low atmospheric pressure, thin and dry air, and a stable daily mean temperature but large temperature difference between day and night, morning or evening and daytime, indoor and outdoor. From the national population census in 2010, the permanent population is about 2.3 billion, and residents of ethnic minorities (eg, Dai, Hui, Yi, Hani and Mongolian minorities) account for $32.27 \%$ of the total population.

\section{Data collection}

Individual records such as age of death, gender, ethnicity, occupation, marital status, cause of death and date of death for all registered deaths for the period 1 January 2009 to 31 May 2016, were obtained from the Yuxi Center for Disease Control and Prevention, which maintains detailed quality assurance and control measures. ${ }^{32} 33$ The underlying causes of death were classified by medical personnel, and examination procedures were routinely performed to ensure accurate data, based on the International Classification of Diseases, 10th revision (ICD-10). Individual data were collapsed into a series of daily counts for the total non-accidental mortality (ICD-10 A00-R99) as well as subcategories by specific cause of death (cardiovascular [I00-I99], heart [I00-I51], stroke [I60-I69] and respiratory disease [J00-J99]), age $(0-64,65-74$ and 75+yearsold), gender (male and female), occupation (farmer and non-farmer), ethnicity (Han nationality and ethnic minorities) and marital status (married and non-married). Daily meteorological data for the same period were obtained from the China Meteorological Data Sharing System, including mean temperature and four other meteorological variables (atmospheric pressure, wind speed, sunshine duration and relative humidity).

\section{Patient and public involvement}

This study is based on daily death number data, which could be obtained from Yuxi Center for Disease Control and Prevention without referral and free of charge. There was no patient and decedent involvement in the presented study.

\section{Statistical analysis}

As daily death number under a Poisson distribution and risk of mortality depend on exposure to temperatures of the current and previous days, ${ }^{24}$ we applied a standard time series quasi-Poisson regression model combined with DLNM to estimate the non-linear and lag effects of mean temperature on mortality, with day of the week, long-term trends, and the four other meteorological variables as potential covariates. This model can capture the complex non-linear relation and lagged effect by combining two functions that define the conventional exposure-response association and the additional lagresponse association. The maximum lag period was set to 28 days to explore the lag structure of temperature effect, and median temperature $\left(17.0^{\circ} \mathrm{C}\right)$ was the reference to calculate attributable risk. ${ }^{31}$ We used natural cubic splines with $7 \mathrm{df}$ per year for time to describe the long-term trends and seasonality and $3 \mathrm{df}$ for the four other meteorological indicators. These model specifications were consistent with previous studies. ${ }^{23} 34$

The total mortality burden attributable to non-reference temperatures can be assessed in terms of fraction 
and number of deaths, and the attributable number can be obtained from the sum of the contributions from all days in the series; its ratio with total number of deaths produces the total attributable fraction. ${ }^{18}$ The overall cumulative relative risk corresponding to each day's temperature was used to compute the attributable fraction and number:

$$
\begin{gathered}
A F_{x, t}=1-\exp \left(-\sum_{l=l_{0}}^{L} \beta_{x_{t-1}, l}\right) \\
A N_{x, t}=A F_{x, t} \times n_{t}
\end{gathered}
$$

where $A F_{x, t}$ and $A N_{x, t}$ are the attributable fraction and the number of cases at day $t(1,2,3 \ldots 2907)$, respectively; $\beta_{x}$ is the risk associated with the exposure to ambient temperatures at level $x$ (ie, $\beta_{x}$; Ref is the referenced temperature; $\beta$ is the coefficient for DLNM of mean temperature; $L$ is the maximum lag for the effect of mean temperature and $n_{t}$ is the observed number of deaths at day $t$.

To estimate the mortality burden from non-accidental deaths, we calculated the total attributable fraction due to the overall temperatures and divided the total effect into exposure to low and high temperatures by summing the subsets corresponding to days with temperatures below and above the median temperature. Also, we explored the mortality burden attributable to mild and extreme temperatures. Extreme cold and heat were defined as temperatures below the 2.5th percentile $\left(5.4^{\circ} \mathrm{C}\right)$ and above the 97.5 th percentile $\left(23.1^{\circ} \mathrm{C}\right)$ of mean temperature, and mild cold and heat were defined as the range between the median temperature and these cut-offs. Monte-Carlo simulations were used to calculate the empirical CIs (eCIs) of the attributable fraction and number, assuming a multivariate normal distribution of the best linear unbiased predictions of the deduced coefficients. ${ }^{185}$ All statistical analyses involved use of R v3.0.3, with the 'dlnm' package to create the DLNM for mean temperature.

\section{RESULTS \\ Descriptive statistics}

We analysed 89467 non-accidental deaths from Yuxi between 2009 and 2016, with an average of 33 deaths per day (range: 12-72). The number of deaths due to cardiovascular disease was 41794 (46.7\%), more than half due to stroke; the proportion due to respiratory disease was $18.5 \%$ (table 1). In individual characteristic subgroups, a higher proportion of deaths was for males, older people ( $\geq 75$ years), people with Han nationality, farmers and

\begin{tabular}{|c|c|c|c|c|c|}
\hline & Total deaths & Min & Median (25th, 75th) & Max & Mean (SD) \\
\hline Total non-accidental & 89467 & 12 & $32(28,38)$ & 72 & $33.0(7.8)$ \\
\hline \multicolumn{6}{|l|}{ Cause-specific } \\
\hline Cardiovascular & 41794 & 2 & $15(12,18)$ & 37 & $15.4(4.9)$ \\
\hline Heart & 17793 & 0 & $6(4,8)$ & 22 & $6.6(3.0)$ \\
\hline Stroke & 22589 & 0 & $8(6,10)$ & 22 & $8.3(3.3)$ \\
\hline Respiratory & 16565 & 0 & $6(4,8)$ & 21 & $6.1(3.1)$ \\
\hline \multicolumn{6}{|l|}{ Age, years } \\
\hline$\leq 64$ & 21678 & 1 & $8(6,10)$ & 19 & $8.0(2.9)$ \\
\hline $65-74$ & 20072 & 0 & $7(5,9)$ & 19 & $7.4(2.9)$ \\
\hline$\geq 75$ & 47717 & 4 & $17(14,21)$ & 43 & $17.6(5.6)$ \\
\hline \multicolumn{6}{|l|}{ Gender } \\
\hline Male & 48939 & 5 & $18(14,21)$ & 43 & $18.1(5.2)$ \\
\hline Female & 40528 & 2 & $15(12,18)$ & 36 & $15.0(4.5)$ \\
\hline \multicolumn{6}{|l|}{ Occupation } \\
\hline Farmer & 68278 & 0 & $7(5,10)$ & 33 & $7.8(3.4)$ \\
\hline Non-farmer & 21189 & 7 & $25(20,30)$ & 57 & $25.2(7.0)$ \\
\hline \multicolumn{6}{|l|}{ Ethnic } \\
\hline Han nationality & 63275 & 6 & $23(19,27)$ & 54 & $23.4(6.4)$ \\
\hline Ethnic minorities & 26192 & 0 & $9(7,12)$ & 24 & $9.7(3.6)$ \\
\hline \multicolumn{6}{|l|}{ Marital status } \\
\hline Married & 54971 & 1 & $12(10,15)$ & 32 & $12.7(4.3)$ \\
\hline Non-married & 34496 & 4 & $20(16,24)$ & 49 & $20.3(5.5)$ \\
\hline
\end{tabular}

Table 1 Daily total non-accidental mortality and by specific causes and individual characteristics in Yuxi, China, 2009-2016

Max, maximum; Min, minimum; 25th, 25th percentile of the distributions; 75th, 75th percentile of the distributions. 


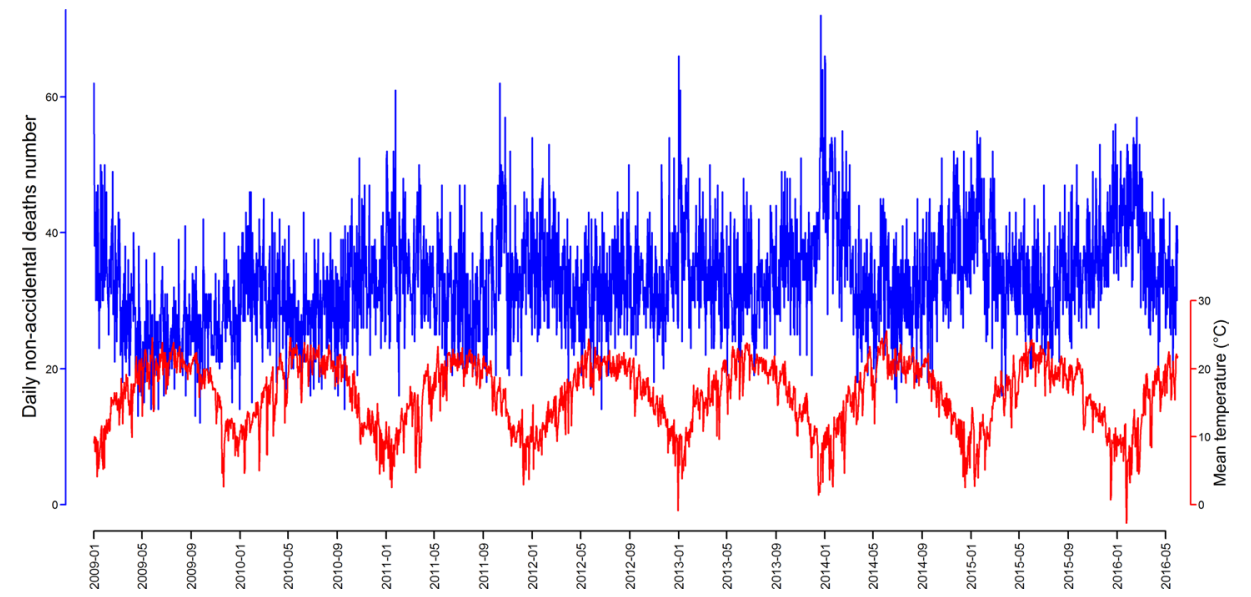

Figure 1 Time series of daily number of non-accidental deaths of Yuxi and mean temperature, 2009-2016.

married people than their corresponding categories. During the study period, the mean daily temperature was $16.1^{\circ} \mathrm{C}$ (ranging from $-3.3^{\circ} \mathrm{C}$ to $25.6^{\circ} \mathrm{C}$ ) (online table $\mathrm{S} 1$ ). The daily number of non-accidental deaths and mean temperature showed an inverse relation (figure 1).

\section{Exposure-response association}

The overall effect of mean temperature on mortality (ie, the total non-accidental deaths and by specific causes and individual characteristics) for lag 0-28days and mean daily temperature distribution are presented in figure 2. In general, the temperature-mortality associations were non-linear and followed slide-shaped curves: the risks due to heat (both mild and extreme) were low and changed slightly (approximately 1 ), whereas the risks due to mild cold and especially extreme cold were increased. The relative risks rapidly increased with decreasing mean temperature. The distribution of mean daily temperature was skewed to the left.

\section{Attributable fraction and number}

Table 2 shows the estimated attributable fraction with $95 \%$ eCIs of daily non-accidental mortality calculated for total and separate components by heat and cold temperatures. For total non-accidental deaths, the attributable fraction was $4.75 \%$ (95\% eCI
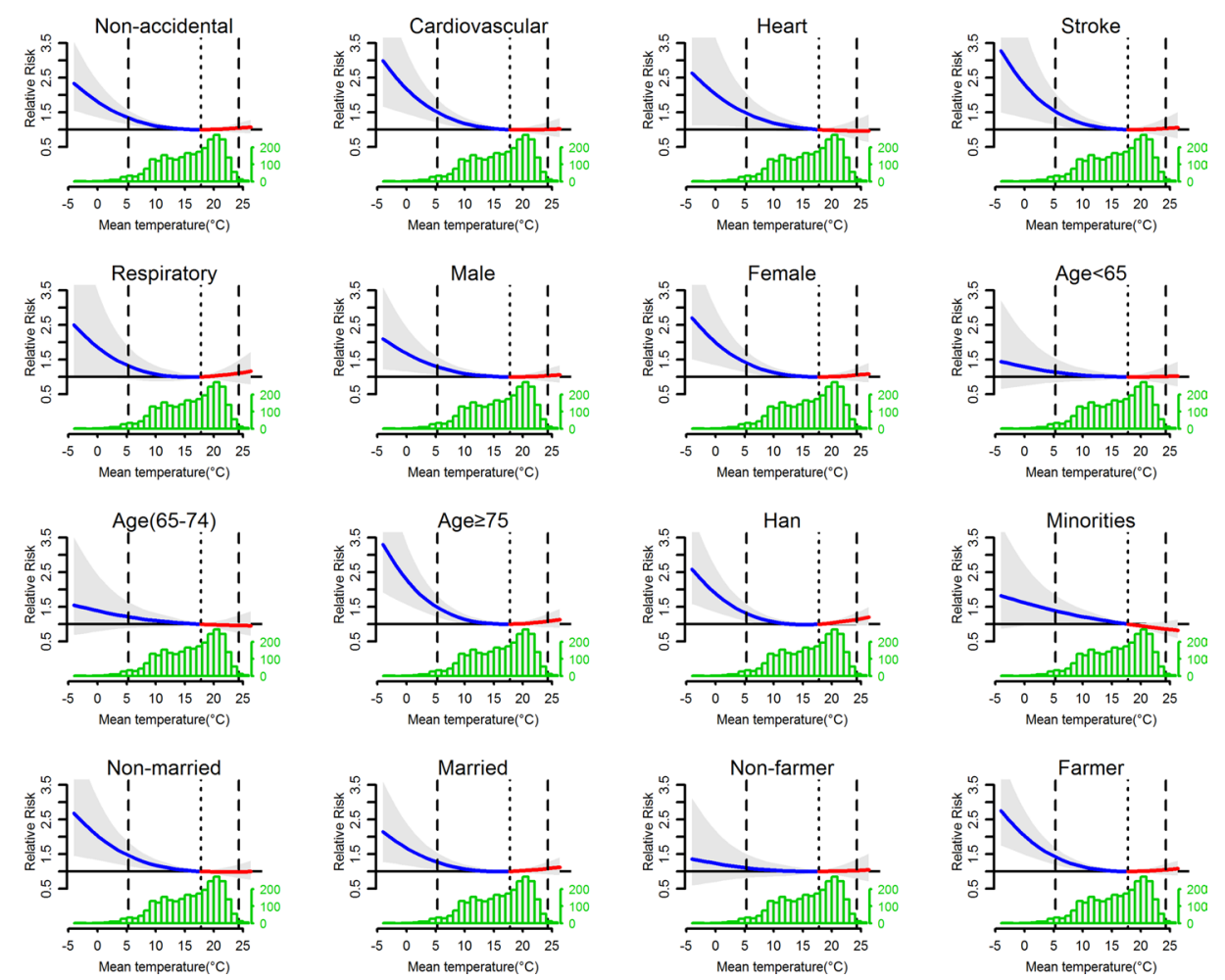

Figure 2 Overall cumulative relative risk (with 95\% empirical Cls, shaded grey) at a lag of 0-28 days in Yuxi, China, with histogram of daily temperature distribution. The dotted lines are the median of the mean temperature, and the dashed lines are the 2.5th and 97.5th percentiles of the distribution of mean temperature. The lines before and after the dotted lines represent the exposure response below (blue lines) and above (red lines) the median of mean temperature, respectively. 
Table 2 Attributable fraction (\%) of total non-accidental mortality and by specific causes and individual characteristics due to mean daily temperature and cold and heat over lag 0-28days in Yuxi, China

\begin{tabular}{|c|c|c|c|}
\hline & Total (\%) & Cold (\%) & Heat (\%) \\
\hline Total non-accidental & 4.75 (2.33 to 6.79$)$ & $4.08(0.86$ to 7.12$)$ & 0.67 (-2.44 to 3.64$)$ \\
\hline \multicolumn{4}{|l|}{ Cause-specific } \\
\hline Cardiovascular & 5.97 (2.74 to 8.74$)$ & 6.18 (1.89 to 10.31$)$ & $-0.21(-5.04$ to 4.33$)$ \\
\hline Heart & $5.25(-0.40$ to 9.57$)$ & $6.48(-0.70$ to 12.47$)$ & $-1.23(-8.59$ to 5.46$)$ \\
\hline Stroke & $6.50(2.22$ to 10.16$)$ & $6.01(-0.11$ to 11.41$)$ & $0.49(-6.18$ to 6.15$)$ \\
\hline Respiratory & $5.42(-0.73$ to 9.71$)$ & $3.50(-5.05$ to 10.95$)$ & $1.93(-5.08$ to 7.90$)$ \\
\hline \multicolumn{4}{|l|}{ Age, years } \\
\hline$\leq 64$ & $1.83(-3.15$ to 5.95$)$ & $1.75(-4.64$ to 7.10$)$ & 0.08 (-6.64 to 5.64$)$ \\
\hline $65-74$ & $2.27(-2.45$ to 6.30$)$ & $3.52(-3.45$ to 9.37$)$ & $-1.25(-7.4$ to 5.01$)$ \\
\hline$\geq 75$ & 6.87 (3.68 to 9.46 ) & 5.34 (0.44 to 9.38$)$ & 1.53 (-2.96 to 5.09$)$ \\
\hline \multicolumn{4}{|l|}{ Gender } \\
\hline Male & 4.16 (0.82 to 7.04$)$ & $3.67(-0.50$ to 7.72$)$ & $0.49(-3.82$ to 4.31$)$ \\
\hline Female & 5.54 (2.18 to 8.31$)$ & 4.66 (0.03 to 9.07$)$ & $0.89(-3.21$ to 5.03$)$ \\
\hline \multicolumn{4}{|l|}{ Occupation } \\
\hline Farmer & 5.66 (3.09 to 7.92$)$ & 4.93 (1.28 to 8.37$)$ & $0.73(-2.7$ to 3.87$)$ \\
\hline Non-farmer & $1.75(-3.58$ to 6.11$)$ & $1.24(-5.77$ to 7.61$)$ & 0.52 (-6.82 to 6.55$)$ \\
\hline \multicolumn{4}{|l|}{ Ethnic } \\
\hline Han nationality & 5.38 (2.44 to 7.96$)$ & $2.79(-1.30$ to 6.49$)$ & $2.59(-0.92$ to 5.80$)$ \\
\hline Ethnic minorities & $2.31(-1.57$ to 6.76$)$ & 6.55 (1.15 to 11.75$)$ & $-4.24(-10.8$ to 1.27$)$ \\
\hline \multicolumn{4}{|l|}{ Marital status } \\
\hline Married & 4.24 (1.17 to 6.99$)$ & $2.74(-1.87$ to 6.53$)$ & $1.50(-2.67$ to 4.83$)$ \\
\hline Non-married & 5.48 (1.69 to 8.56$)$ & 6.10 (1.23 to 10.21$)$ & $-0.61(-5.55$ to 3.95$)$ \\
\hline
\end{tabular}

Results are expressed as attributable fractions (95\% empirical CIS), and the bold values indicate statistical significance.

$2.33 \%$ to $6.79 \%$ ) with the whole temperature range, including heat and cold. Cold temperature was responsible for most of the mortality burden, corresponding to an attributable risk of $4.08 \%(0.86 \%$ to $7.12 \%$ ), whereas the burden due to heat was low and non-significant $(0.67 \% ;-2.44 \%$ to $3.64 \%)$. The attributable risks of cardiovascular and stroke deaths caused by overall temperatures were $5.97 \%(2.74 \%$ to $8.74 \%)$ and $6.50 \%$ (2.22\% to $10.16 \%)$, respectively; the point-estimated risk due to cold was higher for cardiovascular than respiratory deaths $(6.18 \%$ vs $3.50 \%)$.

On stratification by age, the attributable risk due to ambient temperatures increased with age, with attributable fractions of $1.83 \%, 2.27 \%$ and $6.87 \%$ for age $\leq 64$, $65-74$, and $\geq 75$ years, respectively. Those engaged in agriculture had higher attributable fraction, 3.23 times $(5.66 \%$ vs $1.75 \%$ ) due to the overall temperatures and 3.98 times $(4.93 \%$ vs $1.24 \%)$ due to cold, than non-farmers. The estimated burden due to cold was 2.35-fold higher for ethnic minorities than Han nationality ( $6.55 \%$ vs $2.79 \%$ ), whereas the point-estimated attributable fraction caused by the whole temperature range was approximately equal by gender and marital status.
Table 3 displays the estimated mortality fraction attributable to overall temperatures, separated by mild and extreme temperatures. In general, the risk of non-accidental deaths attributable to extreme cold was $1.17 \%$ $(0.58 \%$ to $1.69 \%)$, accounting for a clearly high proportion of $24.6 \%$ of the total mortality burden $(4.75 \%)$ due to the whole temperature range, whereas attributable risks due to mild cold or heat or extreme heat were non-significant. In the cause-specific analyses, the attributable fractions due to extreme cold were $1.57 \%, 1.63 \%$ and $1.49 \%$ for cardiovascular disease, stroke and heart disease, respectively, with no significant association with respiratory disease. The extreme cold-related burden for older people, females, farmers and non-married individuals was slightly higher than their corresponding categories.

Online table S2 presents the attributable number of deaths due to mean temperature, overall and by cold and heat. An estimated number of 4131 non-accidental deaths were due to overall temperatures and 3482 to cold. Online table S3 shows the excess mortality due to extreme and mild cold and mild and extreme heat. Figures 3 and online figure S1 illustrate the daily deaths attributable to cold and heat. The attributable deaths were much larger with cold than heat. 
Table 3 Mortality fraction (\%) attributable to extreme and mild cold and mild and extreme heat by specific causes and individual characteristics

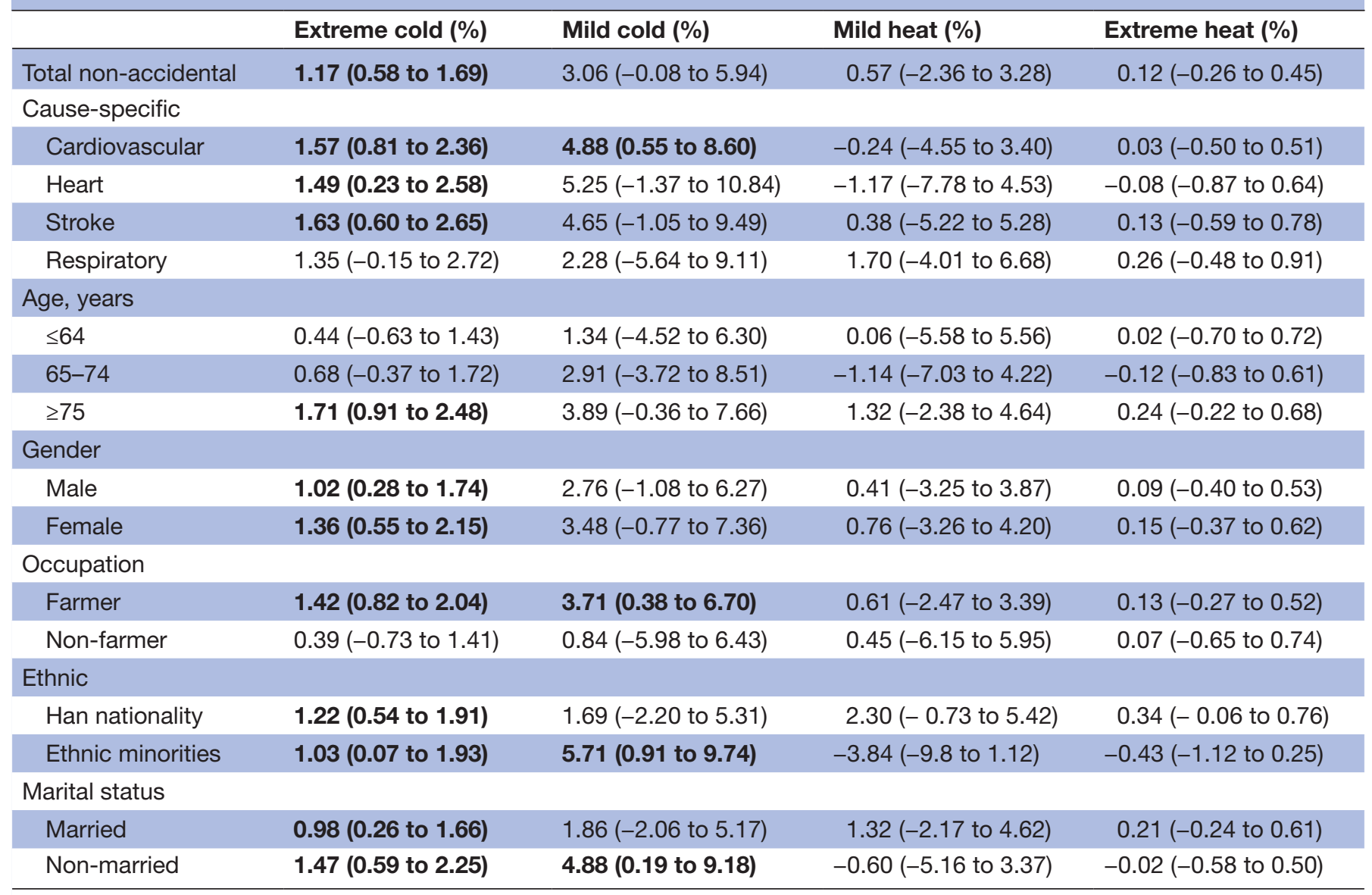

Results are expressed as attributable fractions (95\% empirical Cls), and the bold values indicate statistical significance.

\section{Sensitivity analysis}

Sensitivity analysis to check the stability of our main findings involved changing the maximum lag days $(7,14$ and 21) and the $\mathrm{df}$ of the natural cubic splines for the calendar time (5, 6, 8 and 9 per year) and for the four other meteorological variables one by one $(2,4$ and 5$)$. The attributable fractions for non-accidental mortality due to overall temperatures were relatively robust with sensitivity analyses (online table S4), and the results by causes of deaths

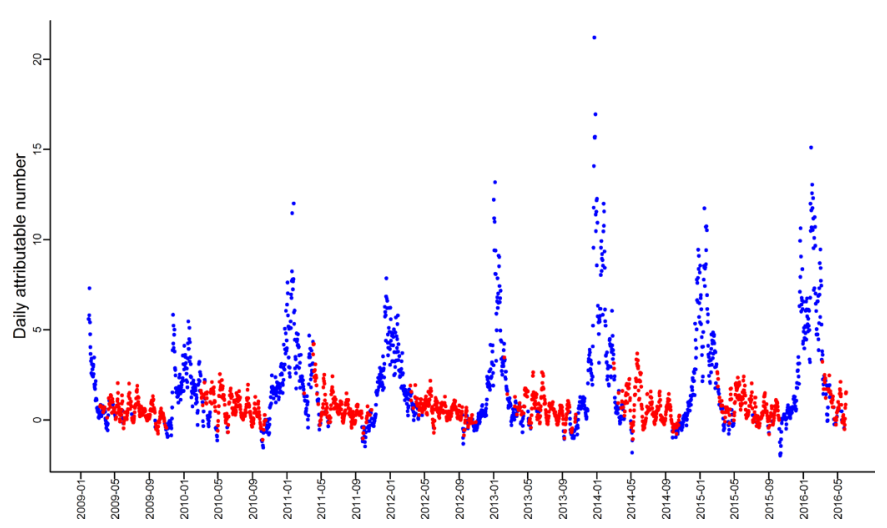

Figure 3 Daily number of total non-accidental deaths attributable to cold (blue points) and heat (red points). and individual characteristics were robust (results not shown).

\section{DISCUSSION}

We quantitatively estimated the attributable risks of non-accidental death and subgroups by specific causes and individual characteristics due to the whole temperature range and to extreme and mild cold and mild and extreme heat for 89467 deaths between 2009 and 2016 in Yuxi, China, a high-altitude region with a unique, subtropical, plateau monsoon climate. The temperature-mortality associations were non-linear and followed slide-shaped curves and the risks rapidly increased with decreasing mean temperature. Excess deaths were attributable to overall temperatures, and cold was responsible for most of the mortality burden. The estimated mortality burden attributable to cold was greater for cardiovascular deaths, older people, farmers, ethnic minorities and non-married individuals than their corresponding categories.

Inconsistent with previous ecological evidences that the temperature-mortality associations were 'U'-or 'V'-shaped curve, with increased mortality risks at extremely low 
and high temperatures, ${ }^{25}$ 36-38 a slide-shaped curve was captured in our study, which shows increased relative risk with low temperature, especially extreme cold but the risk of high temperature changed minimally. The different pattern of temperature-mortality associations might attribute to the unique climate in this high-altitude region. Yuxi city has a distinct subtropical plateau monsoon climate, with four spring-like seasons year-round, giving the city a stable daily mean temperature but large temperature difference between day and night, morning or evening and daytime, indoor and outdoor. Although the city has a stable daily mean temperature of $16.1 \pm 4.9^{\circ} \mathrm{C}$ full year, the daily diurnal temperature range (DTR) was averaging $10.4^{\circ} \mathrm{C}$ (ranging from $1.1^{\circ} \mathrm{C}$ to $21.7^{\circ} \mathrm{C}$ ). Furthermore, we also examined additional non-accidental deaths attributable to ambient temperatures, with larger burden due to cold than heat. A multicountry observational study estimated a total mortality burden of death attributable to non-optimal ambient temperatures; the attributable fraction ranged from $3.37 \%$ in Thailand to $11 \%$ in China, which provides strong evidence for substantial differences between regions or climates. ${ }^{5}$

The cold-related mortality burden is an important public health problem in Yuxi. Findings from our study showed most of the death burden attributable to low temperature and a much lower and non-significant burden due to heat might be owing to unique climatic condition that the differences between minimum and referent temperature was $20.3^{\circ} \mathrm{C}\left(-3.3^{\circ} \mathrm{C}\right.$ vs $\left.17.0^{\circ} \mathrm{C}\right)$ while those between referent and maximum temperature was $8.6^{\circ} \mathrm{C}\left(17.0^{\circ} \mathrm{C}\right.$ vs $\left.25.6^{\circ} \mathrm{C}\right)$. Previous studies have found that most of the mortality burden is caused by exposure to cold days, with comparatively lower attributable risk, or even none, due to heat exposure. For example, Hajat et $a l^{8}$ showed that all-cause mortality attributable to heat ranged from $0.37 \%$ in London (1976-2003) to $1.45 \%$ in Milan (1985-2002), and another study conducted in London from 1986 to 1996 found that attributable fraction of mortality for each $1^{\circ} \mathrm{C}$ decrease below a threshold of $15^{\circ} \mathrm{C}$ was $5.42 \%$ ( $4.13 \%$ to $6.69 \%$ ), with no burden due to heat. ${ }^{39}$ Although extremely low or high temperature corresponded to increased relative risk of mortality, Gasparrini et al found a relatively small part of the death burden attributable to extreme cold temperature, ranging from $0.25 \%$ to $1.06 \%$. Similar results from five East Asian regions showed a 9.36\% mortality burden attributable to overall temperatures, with only $0.80 \%$ due to extreme cold. ${ }^{19}$ However, our current study estimated a larger proportion of attributable mortality fraction due to extreme cold, accounting for about one-quarter of the total mortality burden $(1.17 \%$ vs $4.75 \%)$ although extreme cold days represented only $2.5 \%$ of the whole study period. We found no evidence of additional deaths due to extreme heat in all categories.

Exposure to low temperature has been widely demonstrated to be strongly associated with excess cardiovascular and respiratory deaths, ${ }^{19} 304041$ and the biological processes that underlie cold-related mortality are associated with cardiorespiratory disease. ${ }^{5} 113042$ We found a higher point-estimated attributable risk caused by cold for cardiovascular than respiratory disease deaths. A multicity study including 15 Chinese megacities also identified $15.8 \%$ of the cardiovascular mortality burden due to cold days. ${ }^{25}$ The increased cold-related cardiovascular deaths mainly involved changes in vascular tone, autonomic nervous system response, arrhythmia and oxidative stress. ${ }^{43-45}$ Although we found no evidence for excess burden of respiratory deaths due to cold or heat, other reports have described increased respiratory deaths attributable to ambient temperatures. ${ }^{1046}$ For heart and stroke, the burden of mortality was attributable to only extreme cold, with approximately equivalent values, and other studies found excess heart and stroke deaths attributable to low and/or high temperatures. ${ }^{23} 264047$

Age has been frequently identified as an important modifier of the association between ambient temperatures and human health. ${ }^{1525} 2948$ We found that exposure to cold, particularly extreme cold, was closely related to increased death burden for older than younger people. Several previous surveys found increased age associated with point-estimated attributable risk of cardiovascular mortality and both intracerebral haemorrhage and ischaemic stroke morbidity due to cold, with the highest values in older people. ${ }^{25}{ }^{31}$ Another nation-wide study in Japan found most of the proportion of morbidity burden attributable to days with low temperature in all age groups, with a trend of increasing attributable risk with age: the attributable fraction due to cold was $15.96 \%, 24.84 \%$ and $28.10 \%$ with age $18-64,65-74$, and $75-110$ years, respectively. ${ }^{36}$ Older people were more vulnerable to the temperature effects, mainly because they often have multiple pre-existing chronic conditions and physiological changes in thermoregulation and homoeostasis. ${ }^{9}{ }^{47}$ However, the effect modification of temperature-related mortality by gender has been identified. ${ }^{23293649}$ We observed a higher mortality burden caused by exposure to the cold period among females than males in Yuxi, and the cold-related attributable risk was found higher for females than males in Hanoi, Vietnam, ${ }^{50}$ and in 47 cities in Japan. ${ }^{36}$ The reason for the discrepancy in temperature-related burden by gender might be owing to differences in occupational exposure, physiology and thermoregulation.

A survey in Adelaide, South Australia, provided epidemiological evidence for the impact of heat waves on worker health and safety, which implied that personal occupation might modify the temperature-mortality association. ${ }^{51}$ Our previous studies (Ding $e t a l^{28}{ }^{34}$ ) revealed that farmers were more likely than non-farmers to die on high DTR or cold days, and the present study also showed a higher mortality burden attributable to cold and extreme cold days for farmers than non-farmers. In southwestern China, farmers universally have a poor educational level, disadvantaged socio-economic status and low annual income, which may be linked to poor living conditions, malnutrition and non-access to basic healthcare. In addition, farmers working in the fields may 
have more exposure to ambient temperatures because farming is basically highly related to weather. ${ }^{52}$

A study of nine cities in California found that with each $10^{\circ} \mathrm{F}\left(4.7^{\circ} \mathrm{C}\right)$ increase in mean temperature, the mortality was increased $4.9 \%, 2.5 \%$ and $1.8 \%$ for Blacks, Whites and Hispanics, respectively. ${ }^{29}$ Also, our previous research demonstrated less risk of high DTR associated with non-accidental mortality for the current day for people of Dai ethnic minority than Han nationality. ${ }^{13}$ To our knowledge, no study has estimated the potential effect modification of mortality burden attributable to ambient temperatures by ethnicity. We observed a greater cold- and mild cold-related death for ethnic minorities than Han nationality in Yuxi, which indicated that race/ethnicity may modify the cold-associated mortality burden. We also found lower death burden caused by cold and extreme cold for married people versus those never married, divorced or widowed, possibly because married people can be cared for by their partners during the cold period.

Our study has some limitations. First, the data were from a single city, so generalising the findings to other geographical areas or climates should be cautioned. Second, the data of temperature were from monitoring sites rather than exposure measuring of individual. Third, although the concentration of daily mean $\mathrm{PM}_{10}, \mathrm{NO}_{2}$ and $\mathrm{SO}_{2}$ in Yuxi are much lower than those in other 17 Chinese cities, ${ }^{53}$ we did not control for the potential confounding effects by air pollution due to the unavailability of the complete pollution data in the study area. Last, in the previous multicountry or multicentre studies, ${ }^{52}$ Minimum mortality temperature (MMT) was reasonably used to assess the temperature-mortality associations due to each site corresponding to a MMT. However, inconsistent with those previous studies, our study only involved one city with median temperature as common referent value, which might lead the results incomparable with previous studies. Sensitive analysis with the MMT as referent temperature showed that all of the results were stable substantially when compared with the results estimated by median temperature. But the MMT differed among the subgroups, which led the incomparable results in one city (online table S5).

\section{CONCLUSIONS}

Our study conducted in a high plateau city in southwest China found that most of the death burden is attributable to cold temperature. Our study may have implications for both the research domain and the public health policy arena, which may help policymakers develop intervention strategies to minimise the health effects due to adverse temperatures and predict the climate change impact in this region. Local residents, especially the vulnerable populations such as older people and farmers, need to strengthen their awareness of cold exposure, such as the adaptation of houses (eg, using the air conditioning systems), spending less time outdoors or wearing more clothing when the temperature drops.

Contributors QYZ, ZD and CYD: conceived and designed the experiments. LJL and YFW: provide primary data. PG, SYY, JL, YW and CYD: collected and cleaned the data. CYD: analysed the data and drafted the manuscript. QYZ, ZD and CYD: revised the manuscript and interpreted the results. All authors read and approved the final manuscript.

Funding This study was supported by the Department of Education, Guangdong Government under the Top-tier University Development Scheme for Research and Control of Infectious Diseases (2016016), the National Natural Science Foundation of China (No. 81703323), National key R \& D program of China (2016YFC1304000) and the Scientific Research Program of Health Bureau of Yuxi City (2014).

Competing interests None declared.

Patient consent for publication Not required.

Provenance and peer review Not commissioned; externally peer reviewed.

Data sharing statement Please contact the corresponding author for data requests.

Open access This is an open access article distributed in accordance with the Creative Commons Attribution Non Commercial (CC BY-NC 4.0) license, which permits others to distribute, remix, adapt, build upon this work non-commercially, and license their derivative works on different terms, provided the original work is properly cited, appropriate credit is given, any changes made indicated, and the use is non-commercial. See: http://creativecommons.org/licenses/by-nc/4.0/.

\section{REFERENCES}

1. Watts N, Adger WN, Agnolucci P, et al. Health and climate change: policy responses to protect public health. Lancet 2015;386:1861-914.

2. Lee JY, Kim H. Projection of future temperature-related mortality due to climate and demographic changes. Environ Int 2016;94:489-94.

3. Zhan Z, Zhao Y, Pang S, et al. Temperature change between neighboring days and mortality in United States: a nationwide study. Sci Total Environ 2017;584:1152-61.

4. Song $\mathrm{X}$, Wang $\mathrm{S}, \mathrm{Hu} \mathrm{Y}$, et al. Impact of ambient temperature on morbidity and mortality: an overview of reviews. Sci Total Environ 2017:586:241-54.

5. Gasparrini A, Guo Y, Hashizume M, et al. Mortality risk attributable to high and low ambient temperature: a multicountry observational study. Lancet 2015;386:369-75.

6. Ryti NR, Guo Y, Jaakkola JJ. Global association of cold spells and adverse health effects: a systematic review and meta-analysis. Environ Health Perspect 2016;124:12-22.

7. Guo Y, Gasparrini A, Armstrong BG, et al. Heat wave and mortality: a multicountry, multicommunity study. Environ Health Perspect 2017;125:087006.

8. Hajat S, Armstrong B, Baccini M, et al. Impact of high temperatures on mortality: is there an added heat wave effect? Epidemiology 2006; 17:632-8.

9. Analitis A, Katsouyanni K, Biggeri A, et al. Effects of cold weather on mortality: results from 15 European cities within the PHEWE project. Am J Epidemiol 2008;168:1397-408.

10. Cui $Y$, Yin F, Deng Y, et al. Heat or cold: which one exerts greater deleterious effects on health in a Basin Climate City? Impact of ambient temperature on mortality in Chengdu, China. Int $J$ Environ Res Public Health 2016;13:1225.

11. Tian L, Qiu H, Sun S, et al. Emergency cardiovascular hospitalization risk attributable to cold temperatures in Hong Kong. Circ Cardiovasc Qual Outcomes 2016;9:135-42.

12. Ma W, Wang L, Lin $\mathrm{H}$, et al. The temperature-mortality relationship in China: an analysis from 66 Chinese communities. Environ Res 2015:137:72-7.

13. Ding Z, Li L, Xin L, et al. High diurnal temperature range and mortality: effect modification by individual characteristics and mortality causes in a case-only analysis. Sci Total Environ 2016;544:627-34.

14. Zanobetti A, O'Neill MS, Gronlund CJ, et al. Susceptibility to mortality in weather extremes: effect modification by personal and small-area characteristics. Epidemiology 2013;24:809-19.

15. Basagaña $X$, Sartini $C$, Barrera-Gómez J, et al. Heat waves and cause-specific mortality at all ages. Epidemiology 2011;22:765-72. 
16. Lin H, Liu T, Xiao J, et al. Mortality burden of ambient fine particulate air pollution in six Chinese cities: Results from the Pearl River Delta study. Environ Int 2016;96:91-7.

17. Steenland K, Armstrong B. An overview of methods for calculating the burden of disease due to specific risk factors. Epidemiology 2006;17:512-9.

18. Gasparrini A, Leone M. Attributable risk from distributed lag models. BMC Med Res Methodol 2014;14:55.

19. Lee $\mathrm{WH}$, Lim $\mathrm{YH}$, Dang TN, et al. An investigation on attributes of ambient temperature and diurnal temperature range on mortality in five East-Asian Countries. Sci Rep 2017;7:10207.

20. Yang J, Zhou M, Ou CQ, et al. Seasonal variations of temperaturerelated mortality burden from cardiovascular disease and myocardial infarction in China. Environ Pollut 2017;224:400-6.

21. Chung $\mathrm{Y}$, Lim YH, Honda $\mathrm{Y}$, et al. Mortality related to extreme temperature for 15 cities in northeast Asia. Epidemiology 2015;26:255-62.

22. Guo Y, Gasparrini A, Armstrong B, et al. Global variation in the effects of ambient temperature on mortality: a systematic evaluation. Epidemiology 2014;25:781-9.

23. Yang J, Yin P, Zhou M, et al. The burden of stroke mortality attributable to cold and hot ambient temperatures: Epidemiological evidence from China. Environ Int 2016;92:232-8.

24. Wang C, Zhang Z, Zhou M, et al. Nonlinear relationship between extreme temperature and mortality in different temperature zones: A systematic study of 122 communities across the mainland of China. Sci Total Environ 2017;586:96-106.

25. Yang J, Yin P, Zhou M, et al. Cardiovascular mortality risk attributable to ambient temperature in China. Heart 2015;101:1966-72.

26. Group TE. Cold exposure and winter mortality from ischaemic heart disease, cerebrovascular disease, respiratory disease, and all causes in warm and cold regions of Europe. The Eurowinter Group. Lancet 1997;349:1341-6.

27. Díaz J, Carmona R, Mirón IJ, et al. Comparison of the effects of extreme temperatures on daily mortality in Madrid (Spain), by age group: The need for a cold wave prevention plan. Environ Res 2015;143:186-91.

28. Ding Z, Guo P, Xie F, et al. Impact of diurnal temperature range on mortality in a high plateau area in southwest China: a time series analysis. Sci Total Environ 2015;526:358-65.

29. Basu R, Ostro BD. A multicounty analysis identifying the populations vulnerable to mortality associated with high ambient temperature in California. Am J Epidemiol 2008;168:632-7.

30. Moghadamnia MT, Ardalan A, Mesdaghinia A, et al. Ambient temperature and cardiovascular mortality: a systematic review and meta-analysis. PeerJ 2017;5:e3574.

31. Guo P, Zheng M, Wang Y, et al. Effects of ambient temperature on stroke hospital admissions: results from a time-series analysis of 104,432 strokes in Guangzhou, China. Sci Total Environ 2017;580:307-15.

32. Ma W, Zeng W, Zhou M, et al. The short-term effect of heat waves on mortality and its modifiers in China: an analysis from 66 communities. Environ Int 2015;75:103-9.

33. Lin H, Ratnapradipa K, Wang X, et al. Hourly peak concentration measuring the PM 2.5 -mortality association: results from six cities in the Pearl River Delta study. Atmos Environ 2017;161:27-33.

34. Ding Z, Li L, Wei R, et al. Association of cold temperature and mortality and effect modification in the subtropical plateau monsoon climate of Yuxi, China. Environ Res 2016;150:431-7.
35. Greenland S. Interval estimation by simulation as an alternative to and extension of confidence intervals. Int J Epidemiol 2004;33:1389-97.

36. Onozuka D, Hagihara A. Out-of-hospital cardiac arrest risk attributable to temperature in Japan. Sci Rep 2017;7:39538.

37. Wang X, Li G, Liu L, et al. Effects of extreme temperatures on causespecific cardiovascular mortality in China. Int $J$ Environ Res Public Health 2015;12:16136-56.

38. Yan C, Fei Y, Ying D, et al. Heat or cold: which one exerts greater deleterious effects on Health in a Basin Climate City? Impact of ambient temperature on mortality in Chengdu, China. International Journal of Environmental Research \& Public Health 2016;13:1225.

39. Carson C, Hajat S, Armstrong B, et al. Declining vulnerability to temperature-related mortality in London over the 20th century. Am J Epidemiol 2006;164:77-84.

40. Breitner S, Wolf K, Peters A, et al. Short-term effects of air temperature on cause-specific cardiovascular mortality in Bavaria, Germany. Heart 2014;100:1272-80.

41. Braga AL, Zanobetti A, Schwartz J. The effect of weather on respiratory and cardiovascular deaths in 12 U.S. cities. Environ Health Perspect 2002;110:859-63.

42. Medina-Ramón M, Zanobetti A, Cavanagh DP, et al. Extreme temperatures and mortality: assessing effect modification by personal characteristics and specific cause of death in a multi-city case-only analysis. Environ Health Perspect 2006;114:1331-6.

43. Westfall TC, Yang CL, Chen X, et al. A novel mechanism prevents the development of hypertension during chronic cold stress. Auton Autacoid Pharmacol 2005;25:171-7.

44. Luo B, Shi H, Wang L, et al. Rat lung response to PM2.5 exposure under different cold stresses. Int J Environ Res Public Health 2014:11:12915-26.

45. Tuo B, Li C, Peng L, et al. Analysis of differentially expressed genes in cold-exposed mice to investigate the potential causes of coldinduced hypertension. Exp Ther Med 2014;8:110-4.

46. Zhang Y, Li C, Feng R, et al. The short-term effect of ambient temperature on mortality in Wuhan, China: a time-series study using a distributed lag non-linear model. Int J Environ Res Public Health 2016;13:722-34.

47. Gasparrini A, Armstrong B, Kovats S, et al. The effect of high temperatures on cause-specific mortality in England and Wales. Occup Environ Med 2012;69:56-61.

48. Rocklöv J, Ebi K, Forsberg B. Mortality related to temperature and persistent extreme temperatures: a study of cause-specific and agestratified mortality. Occup Environ Med 2011;68:531-6.

49. Seposo XT, Dang TN, Honda Y. Evaluating the effects of temperature on mortality in Manila City (Philippines) from 2006-2010 using a distributed lag nonlinear model. Int J Environ Res Public Health 2015;12:6842-57.

50. Xuan leTT, Egondi T, Ngoan leT, et al. Seasonality in mortality and its relationship to temperature among the older population in Hanoi, Vietnam. Glob Health Action 2014;7:23115

51. Xiang J, Bi P, Pisaniello D, et al. The impact of heatwaves on workers' health and safety in Adelaide, South Australia. Environ Res 2014;133:90-5

52. Mutekwa VT. Climate change impacts and adaptation in the agricultural sector: the case of smallholder farmers in Zimbabwe. Journal of Sustainable Development in Africa 2009;11.

53. Chen R, Samoli E, Wong C-M, et al. Associations between shortterm exposure to nitrogen dioxide and mortality in 17 Chinese cities: the China Air Pollution and Health Effects Study (CAPES). Environ Int 2012;45:32-8. 